UNIVERSIDADE DE BRASÍLIA

FACULDADE DE MEDICINA

PROGRAMA DE PÓS-GRADUAÇÃO EM MEDICINA TROPICAL

Ana Wieczorek Torrens

EFETIVIDADE DO PROGRAMA BOLSA FAMÍLIA NA CURA DA TUBERCULOSE

BRASÍLIA

2015 


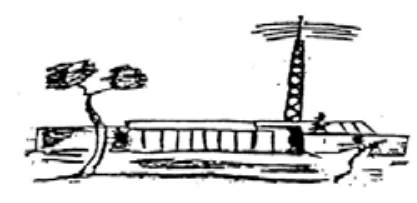

\section{EFETIVIDADE DO PROGRAMA BOLSA FAMÍLIA NA CURA DA TUBERCULOSE}

\section{Ana Wieczorek Torrens}

Dissertação de mestrado apresentada ao Programa de Pós-Graduação em Medicina Tropical da Faculdade de Medicina da Universidade de Brasília para a obtenção do título de mestre em Medicina Tropical, na área de concentração: Epidemiologia e Controle de Doenças Infecciosas e Parasitárias

Orientador: Profo Dr. Mauro Sanchez

\section{BRASÍLIA}

2015 
Ficha catalográfica elaborada automaticamente, com os dados fornecidos pelo(a) autor(a)

We

Wieczorek Torrens, Ana

Efetividade do Programa Bolsa Família na Cura da Tuberculose / Ana Wieczorek Torrens; orientador

Mauro Niskier Sanchez. -- Brasilia, 2015.

$45 \mathrm{p}$.

Dissertação (Mestrado - Mestrado em Medicina Tropical) -- Universidade de Brasilia, 2015.

1. Tuberculose. 2. Transferência de renda condicionada. 3. Adesão ao tratamento. 4. Programa Bolsa Familia. I. Niskier Sanchez, Mauro, orient. II. Título. 
Dedicatória

Dedico essa dissertação ao meu querido chefe Draurio Barreira, idealizador do estudo. 


\section{AGRADECIMENTOS}

Agradeço a toda equipe do Programa Nacional de Controle da Tuberculose que me apoiou enquanto eu estudava.

Agradeço meu orientador Mauro Sanchez pela disponibilidade e companheirismo durante esses dois anos.

Agradeço aos docentes e discentes do Instituto de Saúde Coletiva da Universidade Federal da Bahia pela parceria e discussões no tema.

Agradeço a minha família e amigos pelo orgulho que expressaram no cumprimento dessa etapa da minha vida profissional.

E agradeço especialmente ao pesquisador e amigo Davide Rasella por todo o apoio, em todas as horas que precisei, durante esses dois anos. 


\section{LISTA DE FIGURAS}

Figura 1: Fluxograma de inclusão na população de estudo.............................29 


\section{LISTA DE TABELAS}

Tabela 1: Características demográficas e clínicas da população de estudo segundo exposição ao PBF, Brasil, 2010.

Tabela 2: Características socioeconômicas da população de estudo segundo

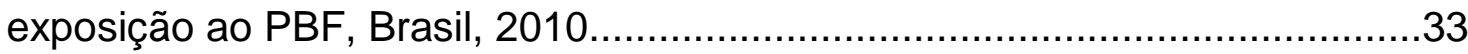

Tabela 3: Desfecho de tratamento da tuberculose segundo exposição ao PBF, Brasil, 2010. .36

Tabela 4: Desfecho de tratamento da tuberculose segundo exposição ao PBF estratificado por realização do tratamento diretamente observado. Brasil, 2010 .38 


\section{LISTA DE ABREVIATURAS}

Aids: Síndrome da Imunodeficiência Humana

Cad.Único: Cadastro Único para Programas Sociais do Governo Federal

CEF: Caixa Econômica Federal

Cl 95\%: Intervalo de confiança 95\%

Coef. Mort.: Coeficiente de Mortalidade

Coef. Inc.: Coeficiente de Incidência

Coef. Prev.: Coeficiente de Prevalência

HIV: Vírus de Imunodeficiência Humana

MDR: Tuberculose multidrogarresistente

MDS: Ministério do Desenvolvimento Social e Combate a Fome

MS: Ministério da Saúde

OMS: Organização Mundial de Saúde

PBF: Programa Bolsa Família

PVHA: Pessoas vivendo com HIV e aids

Sinan: Sistema de Nacional de Agravos de Notificação

TB: Tuberculose

TBDR: Tuberculose droga resistente

TDO: Tratamento Diretamente Observado 


\section{ÍNDICE}

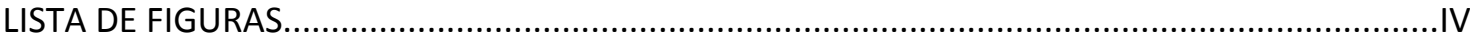

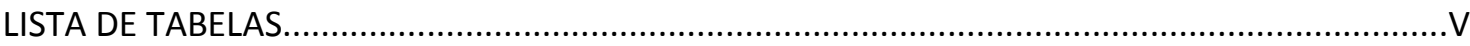

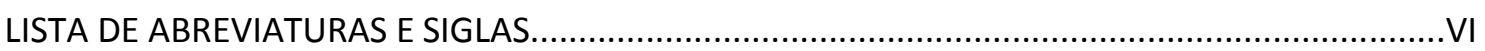

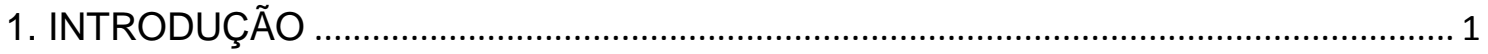

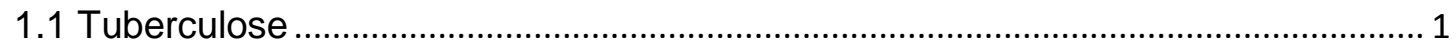

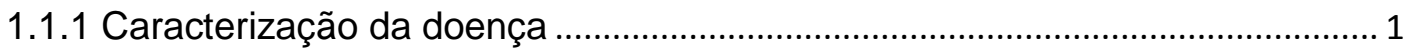

1.1.2 Situação epidemiológica no Brasil e no mundo.................................................. 1

1.2 Determinantes sociais em saúde e da tuberculose ................................................ 3

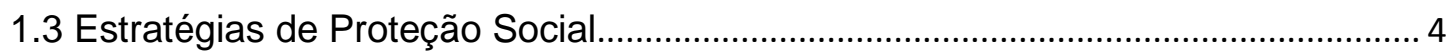

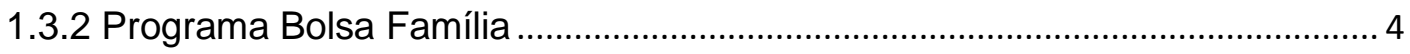

1.3.1 Estratégia pós 2015 de controle da TB........................................................ 5

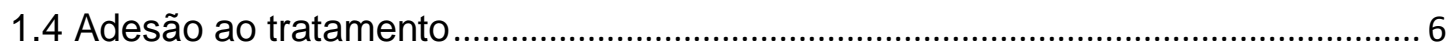

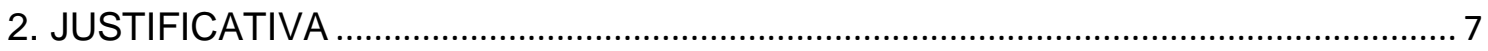

3. OBJETIVOS

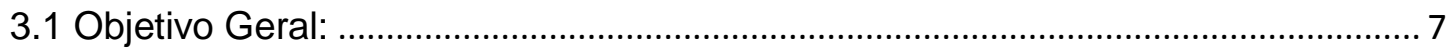

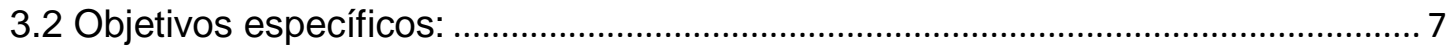

4. MÉTODO

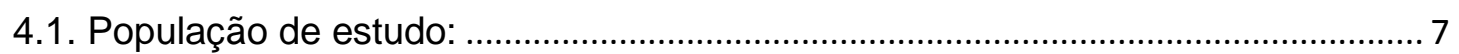

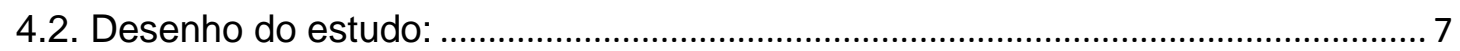

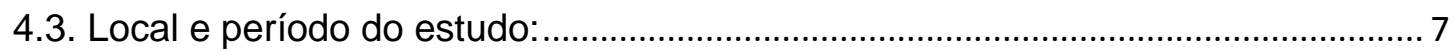

4.4. Coleta dos dados:

4.5. Critérios de inclusão e exclusão:........................................................................... 8

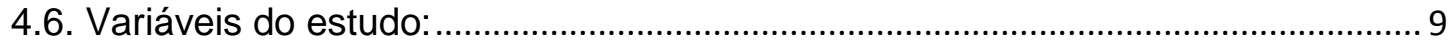

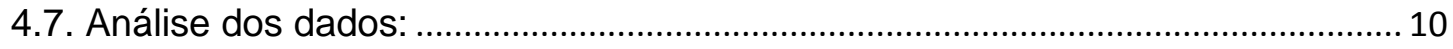

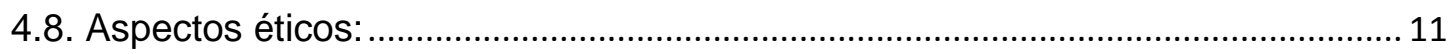


5. RESULTADOS

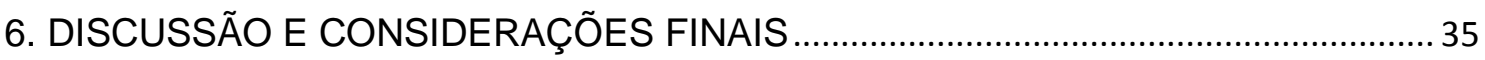

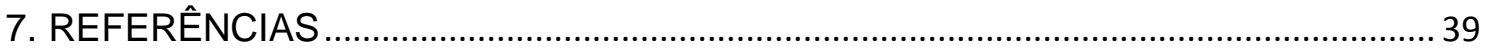

8. ANEXOS

Anexo I: Aprovação no Comitê de Ética .............................................................................. 42 


\section{RESUMO}

TORRENS, Ana. Efetividade do programa bolsa família na cura da tuberculose. Dissertação (Mestrado em Epidemiologia) - Programa de Pós-Graduação em Medicina Tropical, Universidade de Brasília, Brasília 2015.

A tuberculose (TB) é uma das doenças que mais emblematicamente demonstra relação com a pobreza e a exclusão social. O Programa Bolsa Família (PBF) é atualmente o maior programa de transferência de renda condicionada do mundo, que por meio da melhoria das condições materiais e acesso a serviços de saúde tem o potencial de melhorar o acesso a tratamento da TB. O presente estudo tem por objetivo avaliar a efetividade do Programa Bolsa Família na cura da tuberculose. Trata-se de um estudo observacional de coortes históricas. A população de estudo constitui nos casos novos de tuberculose do Brasil diagnosticados expostos e não expostos ao PBF durante o tratamento da TB. Foram estimadas as medidas de associação risco relativo e respectivos intervalos de confiança entre a exposição ao benefício do PBF e o sucesso de tratamento da tuberculose por meio do modelo de regressão de Poisson com variância robusta. A proporção de cura foi $81,7 \%$ entre os expostos ao benefício do PBF durante o tratamento da tuberculose, 5,4 pontos percentuais maior que entre os não expostos durante o tratamento da tuberculose que foi de 76,3\%. A análise multivariada encontrou um risco relativo de 1,07 (Cl 95\%; 1,04-1,11) do benefício do PBF na cura da tuberculose quando ajustado para as covariáveis faixa etária, raça/ cor, forma clínica, diabetes mellitus, sorologia para HIV, tratamento diretamente observado, zona de residência, número de cômodos e material do piso do domicílio, renda per capita familiar e analfabetismo. Os resultados deste estudo mostram como um programa de transferência de renda condicionada pode aumentar a proporção de cura dos pacientes de TB. Este estudo, realizado com a totalidade de pacientes com tuberculose no Brasil elegíveis para o PBF, um dos maiores programas de transferência de renda condicionada do mundo, é relevante porque o sucesso de tratamento continua sendo um grande desafio para 


\section{ABSTRACT \\ Effectiveness of the Bolsa Família Program in tuberculosis cure rate: a retrospective cohort study}

Background: Tuberculosis (TB) is a poverty related disease. The Bolsa Família Program (BFP) is currently the largest conditional cash transfer (CCT) program in the world that targets low income families. By improving the material conditions and access to health services, CCTs have the potential to improve access to TB care and treatment, but no scientific evidence has been established until now. Objective: The study aims to evaluate the effectiveness of the Bolsa Família Program in the tuberculosis cure rate. Methods: An unprecedented record linkage of Brazilian socioeconomic and health datasets has been performed, comparing with a retrospective cohort study design newly diagnosed TB cases exposed and not exposed to conditional cash transfer during TB treatment in the year $2010(n=9,015)$. Adjusted relative risk of tuberculosis cure rate between exposed and not exposed to the BFP was estimated through Poisson regression with robust variance. Results: The cure rate among patients exposed to BFP income transfer was 81.7\%, 5.4 percentage points higher than among those not exposed during tuberculosis treatment. Multivariate regression analysis found a relative risk of $1.07(1,04-$ $1,11)$ of BFP income transfer in tuberculosis treatment success when adjusted for age, race, TB type, diabetes mellitus, HIV status, directly observed treatment (DOT), illiteracy, zone of residence, number of rooms, household floor material, and household per capita income. Conclusions: The results of this study showed - with an innovative record linkage design for a developing country that conditional cash transfer program can contribute to increase TB cure rate. This study, conducted with all TB patients eligible for BFP in Brazil is relevant because treatment success is a major challenge for the country and also social protection for TB patients can be implemented in similar scenarios.

Key words: tuberculosis, conditional cash transfer, adherence to treatment. 


\section{INTRODUÇÃO}

\subsection{Tuberculose}

\subsubsection{Caracterização da doença}

A tuberculose (TB) é uma doença infecciosa e transmissível, causada pelo Mycobacterium tuberculosis. Apesar de qualquer uma das espécies que integram o complexo Mycobacterium tuberculosis causar em a doença, do ponto de vista sanitário, a espécie mais importante é a $M$. tuberculosis. A doença afeta prioritariamente os pulmões, mas pode acometer outros órgãos e sistemas. Em adultos jovens o principal sintoma é a tosse, seca ou produtiva, com expectoração purulenta ou mucoide, com ou sem sangue, e outros sinais e sintomas comuns da tuberculose pulmonar são febre vespertina, sudorese noturna, anorexia e emagrecimento (BRASIL 2014a).

O principal reservatório é o ser humano, e a principal forma de transmissão é aérea. Somente pessoas com tuberculose ativa nas vias aéreas, tuberculose pulmonar ou laríngea, transmitem a doença, por meio de aerossóis produzidos pela tosse e espirro. A forma pulmonar é a mais frequente, representando 85,5\% dos casos novos de tuberculose em 2014 (WHO 2014a), e também a principal responsável pela manutenção da cadeia de transmissão.

Trata-se de uma doença curável em praticamente todos os casos sensíveis aos medicamentos antituberculose, desde que obedecidos os princípios básicos da terapia medicamentosa de associação medicamentosa adequada, doses corretas e uso por tempo suficiente, e a correta operacionalização do tratamento (BRASIL 2014a).

\subsubsection{Situação epidemiológica no Brasil e no mundo}

A tuberculose continua sendo um importante problema de saúde pública no Brasil e no mundo. Segundo o ultimo Relatório Global da Tuberculose da Organização Mundial de Saúde (OMS) estima-se aproximadamente 9 milhões de casos novos de tuberculose por ano no mundo e 1,5 milhões de óbitos relacionados a doença. A doença está presente em todos os continentes, contudo, vinte e dois países concentram aproximadamente $80 \%$ dos casos do mundo (WHO 2014a). 
Ainda de acordo com esse objetivo do milênio de reverter à tendência do coeficiente de incidência (Coef. Inc.) da tuberculose foi alcançado nas seis regiões definidas pela OMS e em quase todos os países de alta carga. Esse apresenta globalmente uma tendência de queda de aproximadamente 1,5\% em média ao ano. Estima-se que os coeficientes de prevalência (Coef. Prev.) e mortalidade (Coef. Mort.) apresentaram redução de $41 \%$ e $45 \%$ respectivamente quando comparados ao ano de 1990, apontando a necessidade de progressos para o alcance das metas propostas pelo Stop-TB Partnership de redução de 50\% desses indicadores até 2015.A região das Américas e do Pacífico Sul já atingiram todas as metas globais para 2015 relacionadas à incidência, prevalência e mortalidade, incluindo o Brasil. As demais regiões ainda precisam aceleras suas tendências de queda para atingir as metas globais de controle da doença (WHO 2014a).

O Brasil faz parte dos 22 países do mundo de alta carga da doença,

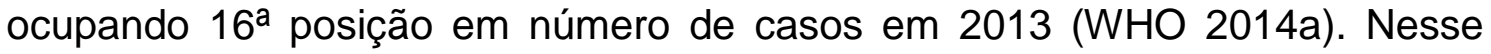
mesmo ano, foram diagnosticados e registrados no Sistema Nacional de Agravos de Notificação (Sinan), aproximadamente 71 mil casos novos de tuberculose no Brasil, representando um Coef. Inc. de aproximadamente 35 casos por 100 mil habitantes. Entretanto a distribuição dos casos não é homogenia entre as unidades da federação (UF), com o Coef. Inc. variando entre 70,6 no Amazonas a 10,8 no Distrito Federal no mesmo ano(BRASIL 2014b).

Nos últimos dez anos foram registrados no Sistema de Informação sobre Mortalidade em torno 4.700 óbitos por tuberculose como causa básica por ano, representando um Coef. Mort. de 2,3 óbitos por 100 mil habitantes no último ano da série, 2012. A mortalidade também não é homogenia entre as UF, variando de 4,2 em Pernambuco a 0,6 no Tocantins nesse mesmo ano (BRASIL 2014b).

O Brasil atingiu todas as metas globais de controle da doença relacionadas à redução da incidência, prevalência e mortalidade, contudo o sucesso de tratamento continua um grande desafio para o país (WHO 2014a). Nos últimos dez anos não houve grande melhora nesse indicador, que se manteve entre 70 a 75\%a nível nacional. Em 2012 a proporção de cura de 
casos novos bacilíferos no país foi em trono de $71 \%$, variando entre 90,4 no Acre a 57,0 no Rio Grande do Norte (BRASIL 2014b).

\subsection{Determinantes sociais em saúde e da tuberculose}

A tuberculose é uma das doenças que mais emblematicamente demonstra relação com a pobreza e a exclusão social (BRASIL 2012)(Hanson 2002).

O adoecimento por tuberculose é influenciado por fatores demográficos, sociais e econômicos, tais como a urbanização crescente e desordenada, a desigualdade na distribuição de renda, moradias precárias e superlotação, a insegurança alimentar, a baixa escolaridade, bem como, dificuldade de acesso aos serviços e bens públicos (Lienhardt 2001) (Blas \& Kurup 2010) (Ximenes et al. 2009).

Sendo assim, a distribuição dos casos de tuberculose ocorre de forma desigual em todo o mundo, concentrando-se nos grupos sociais marginalizados (Hargreaves et al. 2011). No Brasil, afeta principalmente áreas degradadas dos grandes centros urbanos e geralmente está associada à fome, às más condições de moradia e saneamento básico, além de outras condições como o abuso de álcool e outras drogas, e a doenças imunossupressoras, como a aids. Em decorrência das más condições de vida, a dificuldade de acesso aos serviços públicos e até mesmo o comprometimento imunológico certos grupos populacionais são mais vulneráveis à tuberculose (BRASIL 2012). São esses a população indígena, as pessoas em situação de rua, as pessoas privadas de liberdade e aquelas vivendo com HIVIaids (BRASIL 2014a).

O adoecimento por tuberculose, por comprometer de forma significativa a saúde dos indivíduos e suas famílias causa impactos econômicos e sociais (Mauch et al. 2013). Além dos custos vivenciados pelos indivíduos e suas famílias na busca diagnóstica e com tratamento, há a perda de produtividade e consequente redução da renda familiar durante o adoecimento. Os custos indiretos relacionados a perda de produtividade representam a maior parcela no impacto socioeconômico nessas famílias (Mauch et al. 2013). 


\subsection{Estratégias de Proteção Social}

\subsubsection{Programa Bolsa Família}

Programas de transferência de renda são estratégias inovadoras de proteção social. Consistem na concessão de benefício financeiro a indivíduos e famílias de baixa renda ou em situação de vulnerabilidade socioeconômica. E, tem por objetivo a superação da situação de pobreza por meio do fortalecimento do capital financeiro, físico e humano desses indivíduos (Adato \& Hoddinott 2008).

O Programa Bolsa Família (PBF) foi uma das intervenções públicas que mais contribuíram para as mudanças nas condições socioeconômicas da população nos últimos anos (Paim et al. 2011).O PBF foi lançado em 2003, e originou-se da fusão de quatros programas sociais nacionais preexistentes (Soares \& Sátyro 2009). Sua cobertura expandiu muito nos últimos anos, alcançando todos os municípios brasileiros e cobrindo aproximadamente 14,2 milhões de famílias em 2014. Atualmente é o maior programa de transferência de renda condicionada do mundo (BRASIL 2015a).

Todos os beneficiários do PBF devem ser previamente registrados no Cadastro Único para Programas Sociais (Cadúnico) do Ministério do Desenvolvimento Social e Combate à Fome (MDS). Esse identifica e caracteriza as famílias de baixa renda em todo o país e é utilizado para seleção dos beneficiários de todos os programas sociais do governo federal (Lindert et al. 2007).

As transferências de renda do PBF são concedidas as famílias com renda mensal per capta de até $\mathrm{R} \$ 77,00$, independente da sua composição, e as com renda mensal per capta entre $\mathrm{R} \$ 77,01$ e $\mathrm{R} \$ 154,00$ que possuem crianças, adolescentes até 17 anos e gestantes ou lactantes. Os benefícios monetários variam de acordo com a renda e a composição familiar e o pagamento mensal ocorre preferencialmente à mulher quando houver (Lindert et al. 2007). Para receber os benefícios as famílias devem cumprir com condicionalidades relacionadas à educação e à saúde. Para acompanhamento das condicionalidades de saúde, todas as famílias estão vinculadas a uma unidade básica de saúde (BRASIL 2009). 


\subsubsection{Estratégia pós 2015 de controle da TB}

O reconhecimento da complexidade dos determinantes das doenças relacionadas à pobreza evoca um maior esforço na construção de intervenções intersetoriais que enfrentem de forma mais abrangente os problemas de saúde da população, especialmente dos grupos mais pobres (Savioli et al. 2010).

A nova estratégia de controle da tuberculose pós-2015, lançada pela OMS em 2014 e intitulada "Estratégia de Eliminação da Tuberculose"(WHO 2015), possui uma visão mais ampliada e metas que contribuam para a agenda global pós-2015 de saúde e desenvolvimento. É baseada em três pilares: i. Prevenção e cuidado integral centrado no paciente, ii. Políticas arrojadas e sistemas de proteção, e iii. Pesquisa e inovação. Nela são consideradas ações de prevenção, controle e cuidados de tuberculose, envolvendo assim a participação de líderes nacionais dos programas de saúde pública para fazer face aos problemas enfrentados pelas comunidades afetadas em gestão de sistemas de saúde, em proteção social e desenvolvimento econômico e social, e no âmbito da pesquisa (WHO 2015).

O segundo pilar, dedicado ao compromisso político, participação social, cobertura universal e proteção social dos pacientes com tuberculose, propõe intervenções socioeconômicas para prevenir e controlar a doença e também recomenda estratégias de proteção social para os pacientes com tuberculose. Pretende também eliminar o estigma e a discriminação associados a certos grupos populacionais e que se constituem como barreiras históricas as políticas públicas. Além disso, possui como uma de suas metas a eliminação dos custos catastróficos relacionados ao adoecimento por tuberculose (WHO 2015).

No Brasil, em consonância com a nova estratégia de controle da tuberculose, foi lançada a Resolução n. ${ }^{0}$ 444/2011 (BRASIL 2011), do Conselho Nacional de Saúde, a qual preconiza a articulação dos programas de controle da tuberculose com as demais políticas públicas a fim de desenvolver ações que considerem as necessidades específicas, sobretudo das populações mais vulneráveis (BRASIL 2014b). A intersetorialidade torna-se uma prioridade no enfrentamento da doença no país.

As inequidades sociais dificultam os avanços no controle da doença (Lönnroth et al. 2010), e nesse sentido o sucesso do futuro da eliminação da 
tuberculose pode depender do progresso em diversas áreas afins em conjunto (Jaramillo E 1999).

\subsection{Adesão ao tratamento}

A tuberculose é uma doença de longo tratamento e difícil adesão. $O$ tratamento da tuberculose sensível aos medicamentos de primeira linha tem duração mínima de seis meses e por isso é fundamental que o profissional de saúde acolha o usuário, desde o diagnóstico até sua alta. A abordagem humanizada e o estabelecimento de um forte vínculo entre profissional de saúde e usuário auxiliam na adesão ao tratamento (BRASIL 2014a).

O tratamento deve ser desenvolvido sob regime ambulatorial e realizado preferencialmente na atenção básica e o paciente deve ser orientado de forma clara quanto às características clínicas da tuberculose e do tratamento a que será submetido e para as possíveis consequências do uso irregular dos medicamentos e eventos adversos (BRASIL 2014a).

Apesar do diagnóstico e tratamento serem garantidos pelos SUS, na busca da confirmação diagnóstica e durante o tratamento, os pacientes e suas famílias experimentam custos diretos médicos e não médicos, principalmente relacionados complementação alimentar e transporte, e custos indiretos, devido à perda de produtividade, o que leva a um ciclo de empobrecimento (Mauch et al. 2013). Esse quadro dificulta a adesão ao tratamento e leva a desfechos desfavoráveis como abandono, falência de tratamento, internação, tuberculose drogaresistente e óbito.

Considerando ainda a discriminação sofrida por certos grupos populacionais mais vulneráveis a tuberculose, são necessárias medidas específicas para garantir o acesso aos serviços de saúde e qualificar o cuidado que esses recebem para garantir o sucesso de tratamento. Para tanto, torna-se importante a articulação com diversos atores governamentais, não governamentais e Instituições de Ensino e Pesquisa (BRASIL 2014b).

Por ser uma doença de grande magnitude, a tuberculose está na agenda de prioridades do Ministério da Saúde (MS), e considerando a determinação social da doença, o acesso das populações mais vulneráveis aos serviços de saúde e a proteção social durante o tratamento são prioridades programáticas. 


\section{JUSTIFICATIVA}

Há poucas avaliações de impacto do Programa Bolsa Família na saúde, e até o momento não há dados do seu efeito no controle da tuberculose. Os resultados do presente estudo poderão subsidiar os programas de controle da tuberculose na implantação de estratégias combinadas de ações programáticas de controle da doença e de proteção social específicas para pacientes com tuberculose.

\section{OBJETIVOS}

\subsection{Objetivo Geral:}

Avaliar a efetividade do Programa Bolsa Família na cura da tuberculose.

\subsection{Objetivos específicos:}

- descrever as características demográficas, clínicas e socioeconômicas dos pacientes com tuberculose beneficiários do Programa Bolsa Família;

- Avaliar a efetividade do Programa Bolsa Família na cura da tuberculose.

\section{MÉTODO}

4.1. População de estudo: A população de estudo consiste nos casos novos de tuberculose (CID10: A15-A19) (WHO 1998) do Brasil notificados no Sistema Nacional de Agravos de Notificação (Sinan) do Ministério da Saúde e registrados no Cad.Único e inclusos na folha de pagamento do PBF da Caixa Econômica Federal (CEF).

\subsection{Desenho do estudo:}

Trata-se de um estudo observacional de coortes históricas.

4.3. Local e período do estudo: Brasil, todos os meses do ano diagnóstico 2010. 
- Sinan: informações sobre os casos durante o tratamento da tuberculose que possui duração de seis a nove meses;

- Cadúnico: informações sobre os casos resgatas no cadastro no período de2001 a novembro de 2011;

- Folha de pagamento do PBF: informações sobre o benefício do PBF recebido pelos casos em novembro de 2011 e data de competência inicial do beneficio.

4.4. Coleta dos dados: Os dados foram obtidos de um banco de dados construído a partir do relacionamento das bases de dados nacionais do Sinan, do Cad.Único e da folha de pagamento mensal do PBF.

O relacionamento das bases de dados foi realizado pela Secretaria Nacional de Renda e Cidadania do Ministério do Desenvolvimento Social e Combate à Fome (Senarc - MDS), e consistiu em:

- pareamento determinístico entre os casos de tuberculose notificados no Sinan diagnosticados em 2010 e ao mesmo tempo ativos no registro do Cadastro Único para Programas Sociais (Cad.Único) do Governo Federal - com informações desta base de dados no período de 2001 a novembro de 2011. Utilizou-se como variáveis de identificação: data de nascimento, nome completo, nome da mãe e soundex de ambos, após manipulação dessas.

- pareamento determinístico entre o Cad.Único e a folha de pagamento do PBF de novembro de 2011, a partir do número de Identificação Social (NIS) para identificar os indivíduos registrados no CadÚnico beneficiários do PBF.

Após ambos os relacionamentos das bases de dados, encontrou-se um pareamento de $13,1 \%$ (9.414) dos casos notificados de tuberculose.

\subsection{Critérios de inclusão e exclusão:}

Foram incluídos todos os casos novos de tuberculose notificados no Sinan, e registrados e ativos no Cadúnico, e presentes na folha de pagamento do PBF.

Escolheu-se incluir na população de estudo apenas os casos novos que tivessem no Cad.Único e na folha de pagamento do PBF para ter um grupo de 
comparação que fossem quanto mais similares possíveis a contrafactuais (World Bank 2010).Trata-se de um grupo com as mesmas características dos pacientes expostos ao benefício do PBF - ou seja elegíveis para receber os benefícios - mas não inclusos ainda no programa durante o tratamento da TB, devido ao próprio processo de expansão do programa que incluí busca ativa de famílias em situação de vulnerabilidade, atrasos no registro no Cad.Único e até mesmo atrasos administrativos na concessão dos benefícios.

- Grupo exposto: casos novos de TB beneficiários do PBF durante o período de acompanhamento do tratamento. Esses consistem nos casos com data de competência inicial do benefício do PBF anterior à data de encerramento do caso no Sinan.

- Grupo não exposto: casos novos de TB, elegíveis para receber o beneficio do PBF, contudo com início de recebimento do benefício do PBF após o encerramento do acompanhamento do tratamento. Esses consistem nos casos com data de competência inicial do benefício do PBF posterior à data de encerramento do caso no Sinan.

Foram considerados casos novos de TB todos aqueles com tipo de entrada no sistema de informação como caso novo ou história pregressa desconhecida $(n=23)$. Foram excluídos todos os indivíduos que: apresentaram mudança de diagnóstico durante o tratamento da TB ( $n=1.600)$; sem informação da data de encerramento do tratamento $(n=359)$ ou mês de competência inicial do benefício do PBF $(n=15)$, e diagnosticados com tuberculose multidrogarresistente (MDR) $(n=25)$.

4.6. Variáveis do estudo: As variáveis analisadas no estudo foram:

Sinan:

- Dados demográficos e clínicos: sexo, idade, raça/ cor, data do diagnóstico, forma clínica, resultado do exame para HIV, diabetes, alcoolismo; tratamento diretamente observado (TDO) e desfecho de tratamento.

Cadúnico: 
- Identificação e controle da pessoa e da família: data de cadastramento, relação de parentesco com o responsável da família, valor da renda per capta familiar;

- Características do domicílio: zona de residência, número de cômodos, material do piso, material da casa, água canalizada, abastecimento de água, banheiro, escoamento sanitário e destino do lixo;

- Escolaridade e trabalho e remuneração: alfabetização.

Folha de pagamento da CEF: valores dos benefícios básico, variável para crianças e adolescentes até 16 anos, variável para gestantes e nutrizes, variável vinculado ao adolescente e variável de caráter extraordinário; e mês e ano de competência inicial do benefício.

4.7. Análise dos dados: A análise seguiu as seguintes etapas.

- Estatística descritiva: foram avaliadas a distribuição, as características das variáveis, a completitude e a consistência dos dados. A variável número de cômodos no domicílio teve quatro observações com número de cômodos acima de onze imputados com o valor de quatro cômodos. As variáveis data de diagnóstico, tipo de entrada, idade, raça/ cor, forma clínica, diabetes mellitus, teste para HIV, TDO, número de cômodos, material do piso, material das paredes, abastecimento de água, escoamento sanitário, coleta de lixo, iluminação do domicílio e renda per capta familiar foram recategorizadas, seguindo a distribuição da variável na população de estudo, ou segundo critérios encontrados na literatura para dicotomização das variáveis em questão. Foi considerado como TDO não realizado os casos com registro de não realização e sem informação. Os resultados de teste para HIV em andamento e não realizado foram considerados como sorologia desconhecida, e esses foram incluídos como uma categoria na análise. Foi considerado como piso inapropriado aqueles de terra, madeira aproveitada ou outro material não especificado. As características clínicas, epidemiológicas, demográficas e socioeconômicas foram utilizadas como descritoras da população do estudo, e as variáveis foram apresentadas como frequências absolutas e relativas (proporções), e medianas e intervalos interquartílicos se discretas ou 
contínuas. Foram utilizados testes de diferenças de proporções e medianas: teste de diferença de proporções, teste qui quadrado e Wilcoxon rank-sum test para avaliar diferenças entre subpopulações.

- Análise multivariada:

- Variável dependente: sucesso de tratamento. Compreende os casos de cura comprovada, que são aqueles com um exame de baciloscopia de escarro negativa no quinto ou sexto mês de tratamento e mais um entre os quatro primeiros meses, e também os casos de tratamento completo sem comprovação segundo critério descrito acima. Foram considerados como não sucesso de tratamento os demais desfechos: abandono, óbito por e com TB, transferência e ignorado,

- Variável independente: exposição ao benefício do PBF. Compreende os casos beneficiários de qualquer uma das partes que compõe o benefício de transferência de renda do programa.

Foram avaliados potenciais confundidores e foram incluídos no modelo multivariado as variáveis com completitude acima de 80\% e significância estatística na análise bivariada. As variáveis forma clínica e sorologia para HIV foram incluídas devido a constatação da sua associação com desfecho e exposição na literatura independe do critério acima. Foram estimadas as medidas de associação risco relativo e respectivos intervalos de confiança entre a exposição ao benefício do PBF e o sucesso de tratamento da tuberculose por meio do modelo de regressão de Poisson com variância robusta (Newton et al. 2010). Tal modelo é adequado para estimar risco relativo em estudos de coorte de desfechos comuns, como no presente estudo no qual a proporção de cura é superior a 70\% (McNutt et al. 2003). Para examinar uma possível modificação do efeito do PBF no sucesso e tratamento segundo a realização de TDO, também foi aplicado esse mesmo modelo após estratificação da base de dados segundo realização do TDO.

Os dados foram analisados nos softwares Stata v.12 (StataCorp, College Station, Texas USA).

4.8. Aspectos éticos: O projeto foi submetido e aprovado pelo Comitê de Ética em pesquisa do Centro de Ciências da Saúde da Universidade Federal do Espírito Santo, parecer número 242.831. No que tange aos aspectos éticos 
foram seguidos os critérios estabelecidos pelo Comitê de Ética e Pesquisa com Seres Humanos conforme a Resolução 466/12 do Conselho Nacional de Saúde. A confidencialidade de todas as informações constantes nos instrumentos de pesquisa foram asseguradas em todas as etapas da investigação e a publicação dos resultados ocorrerá de maneira tal que os sujeitos jamais serão identificados.

\section{RESULTADOS}

\section{EFETIVIDADE DO PROGRAMA BOLSA FAMÍLIA NA CURA DA TUBERCULOSE}

Torrens, ${ }^{1}$; Sanchez, M. ${ }^{1} ;$ Maciel E. ${ }^{2} ;$ Nery, J.. ${ }^{3}$ Draurio, B ${ }^{4}$. Rasella, D ${ }^{3}$

1. Universidade de Brasília; 2. Universidade Federal do Espírito Santo; 3.

Universidade Federal da Bahia 4.Ministério da Saúde do Brasil

\section{Resumo}

Introdução: A tuberculose (TB) é uma das doenças que mais emblematicamente demonstra relação com a pobreza e a exclusão social. O Programa Bolsa Família (PBF) é atualmente o maior programa de transferência condicionada do mundo que por meio da melhoria das condições materiais e acesso a serviços de saúde têm o potencial de melhorar o acesso a tratamento de qualidade da TB. Objetivo: Avaliar a efetividade do Programa Bolsa Família na cura da tuberculose. Método: Estudo observacional de coortes históricas. A população de estudo constitui nos casos novos de tuberculose do Brasil diagnosticados expostos e não expostos ao PBF durante o tratamento da TB. Foram estimadas as medidas de associação risco relativo e respectivos intervalos de confiança entre a exposição ao benefício do PBF e o sucesso de tratamento da tuberculose por meio do modelo de regressão de Poisson com variância robusta. Resultados: a proporção de cura foi $81,7 \%$ entre os expostos ao benefício do PBF durante o tratamento da tuberculose, 5,4 pontos percentuais maior que entre os não expostos durante o tratamento da tuberculose que foi de 76,3\%. A análise multivariada encontrou um risco relativo de 1,07 ( $\mathrm{Cl} 95 \% ; 1.04$ - 1,07) do benefício do PBF na cura da 
tuberculose quando ajustada para as covariáveis faixa etária, raça/ cor, forma clínica, diabetes mellitus, sorologia para HIV, tratamento diretamente observado, zona de residência, número de cômodos e material do piso do domicílio, renda per capta familiar e analfabetismo. Conclusão: Os resultados deste estudo mostram como um programa de transferência de renda condicionada pode aumentar a proporção de cura dos pacientes de TB. Este estudo, realizado com a totalidade de pacientes com tuberculose no Brasil elegíveis para o PBF, um dos maiores programas de transferência de renda condicionada do mundo, é relevante porque o sucesso de tratamento continua sendo um grande desafio para o país.

Palavras-chave: tuberculose, transferência de renda condicionada, adesão ao tratamento da tuberculose. 


\section{Introdução}

A tuberculose é uma das doenças que mais emblematicamente demonstra relação com a pobreza e a exclusão social (BRASIL 2014b) (Hanson 2002). A distribuição dos casos de tuberculose ocorre de forma desigual em todo o mundo, concentrando-se nos grupos sociais marginalizados (Hargreaves et al. 2011). No Brasil afeta, principalmente, áreas degradadas dos grandes centros urbanos e geralmente está associada à fome, às más condições de moradia e saneamento básico, além de outras condições como o abuso de álcool e outras drogas, e a doenças imunossupressoras, como a aids (BRASIL 2012).

O Brasil faz parte dos 22 países do mundo de alta carga da doença, ocupando 16a posição em número de casos em 2013 (WHO 2014a).Nesse mesmo ano, foram diagnosticados e registrados no Sistema Nacional de Agravos de Notificação (Sinan), aproximadamente 71 mil casos novos de tuberculose no Brasil, representando um Coeficiente de Incidência de aproximadamente 35 casos por 100 mil habitantes.

O reconhecimento da complexidade dos determinantes das doenças relacionadas à pobreza evoca um maior esforço na construção de intervenções intersetoriais que enfrentem de forma mais abrangente os problemas de saúde da população, especialmente dos grupos mais pobres (Savioli et al. 2010).

As inequidades sociais dificultam os avanços no controle da doença (Lönnroth et al. 2010), e nesse sentido o sucesso do futuro da eliminação da tuberculose pode depender do progresso em diversas áreas afins em conjunto (Jaramillo E 1999). Programas de transferência de renda são estratégias inovadoras de proteção social e superação da situação de pobreza (Adato \& Hoddinott 2008), que por meio da melhoria das condições materiais, psicossociais e acesso a serviços de saúde, em princípio têm o potencial de reduzir a vulnerabilidade ao adoecimento e melhorar o acesso a tratamento de qualidade da TB(Boccia et al. 2011).

O Programa Bolsa Família (PBF) foi uma das intervenções públicas que mais contribuíram para as mudanças nas condições socioeconômicas da população nos últimos anos (Paim et al. 2011). Lançado em 2003,e é atualmente o maior programa de transferência de renda condicionada do 
mundo (Soares \& Sátyro 2009). Sua cobertura expandiu muito nos últimos anos, alcançando todos os municípios brasileiros, cobrindo aproximadamente 14,2 milhões de famílias em 2014 (BRASIL 2015a).

Todos os beneficiários do PBF devem ser previamente registrados no Cadastro Único para Programas Sociais (CadÚnico) do Ministério do Desenvolvimento Social e Combate à Fome (MDS). Esse identifica e caracteriza as famílias de baixa renda em todo o país e é utilizado para seleção dos beneficiários de todos os programas sociais do governo federal (Lindert et al. 2007).As transferências de renda do PBF são concedidas as famílias com renda mensal per capta de até $R \$ 77,00$, independente da sua composição, e as com renda mensal per capita entre $R \$ 77,01$ e $R \$ 154,00$ que possuem crianças, adolescentes até 17 anos e gestantes ou lactantes. Os benefícios monetários variam de acordo com a renda e a composição familiar e o pagamento mensal ocorre preferencialmente à mulher quando houver (Lindert et al. 2007) e para receber os benefícios as famílias devem cumprir com condicionalidades relacionadas à educação e à saúde. Para acompanhamento das condicionalidades de saúde todas as famílias estão vinculadas a uma unidade básica de saúde (BRASIL 2009).

Há poucas avaliações de impacto do Programa Bolsa Família na saúde, e até o momento não há dados do seu efeito no controle da tuberculose. Os resultados do presente estudo poderão subsidiar os programas de controle da tuberculose na implantação de estratégias combinadas de ações programáticas de controle da doença e de proteção social específicas para pacientes com tuberculose.

O objetivo do presente estudo é avaliar a efetividade do Programa Bolsa Família no sucesso de tratamento da tuberculose

\section{Método}

Trata-se de um estudo observacional de coortes históricas. A população de estudo constitui nos casos novos (CID10: A15-A19) (WHO 1998)de tuberculose do país diagnosticados em todos os meses de 2010 e notificados no Sistema Nacional de Agravos de Notificação (Sinan) e ao mesmo tempo ativos no registro do Cadastro Único para Programas Sociais do Governo Federal - com informações desta base de dados no período de 2001 a 
novembro de 2011 - e estivessem também incluídos na folha de pagamento do PBF da Caixa Econômica Federal (CEF) nesse último mês.

Os dados foram obtidos de um banco de dados construído a partir do relacionamento das bases de dados nacionais do Sinan, do Cad.Único e da folha de pagamento mensal do PBF da Caixa Econômica Federal. O relacionamento foi realizado pela Secretaria Nacional de Renda e Cidadania do Ministério do Desenvolvimento Social e Combate à Fome (Senarc - MDS), e consistiu nos pareamentos determinístico entre o Cad.Único e os casos de tuberculose notificados no Sinan diagnosticados em 2010, e a folha de pagamento do PBF.O pareamento foi realizado utilizando como variáveis de identificação: data de nascimento, nome completo, nome da mãe e soundex de ambos, após manipulação desses; e a partir do número de Identificação Social (NIS) para identificar os indivíduos registrados no Cadúnico beneficiários do PBF. Encontrou-se um pareamento de 13,1\% (=9.414).

Foram incluídos todos os casos novos de tuberculose notificados no Sinan, e registrados e ativos no Cadúnico, e presentes na folha de pagamento do PBF. Escolheu-se incluir na população de estudo apenas os casos novos que tivessem no Cad.Único e na folha de pagamento do PBF para ter um grupo de comparação que fossem quanto mais similares possíveis a contrafactuais (World Bank 2010).Trata-se de um grupo com as mesmas caraterísticas dos casos expostos ao benefício do PBF - ou seja elegíveis para receber os benefícios - mas não inclusos ainda no programa durante o tratamento da TB, devido ao próprio processo de expansão do programa que incluí busca ativa de famílias em situação de vulnerabilidade, atrasos no registro no Cad.Único e até mesmo atrasos administrativos na concessão dos benefícios.

O grupo de expostos foi composto casos novos de TB beneficiários do PBF durante o período de acompanhamento do tratamento, com data de competência inicial do benefício do PBF anterior a data de encerramento do caso no Sinan. O grupo de não expostos foi composto pelos casos novos de TB, elegíveis para receber o beneficio do PBF, contudo com início de recebimento do benefício do PBF após o encerramento do acompanhamento do tratamento. Esses consistem nos casos com data de competência inicial do 
benefício do PBF posterior a data de encerramento do caso no Sinan. Foram excluídos todos os indivíduos que: apresentaram mudança de diagnóstico durante o tratamento da TB $(n=1.600)$; não possuíam data de encerramento do tratamento $(n=359)$ ou mês de competência inicial do benefício do PBF informado $(n=15)$, e diagnosticados com tuberculose multidrogarresistente (MDR) $(n=25)$.

$\mathrm{Na}$ análise descritiva foram avaliada a distribuição, as características das variáveis, a completitude e a consistência dos dados. As variáveis data de diagnóstico, tipo de entrada, idade, raça/ cor, forma clínica, diabetes mellitus, teste para HIV, tratamento diretamente observado (TDO), número de cômodos, material do piso, material das paredes, abastecimento de água, escoamento sanitário, coleta de lixo, iluminação do domicílio e renda familiar per capta foram recategorizadas seguindo a distribuição da variável na população de estudo, ou segundo critérios encontrados na literatura para dicotomização das variáveis em questão. Foi considerado como TDO não realizado os casos com registro de não realização e sem informação. As características clínicas, epidemiológicas, demográficas e socioeconômicas foram utilizadas como descritoras da população do estudo, e as variáveis foram apresentadas como frequências absolutas e relativas (proporções), e medianas e intervalos interquartílicos se discretas ou contínuas. Foram utilizados os testes de diferenças de proporções e medianas: teste de diferença de proporções, teste qui quadrado e Wilcoxon rank-sum test para avaliar diferenças entre subpopulações.

$\mathrm{Na}$ análise multivariada, usou-se como variável dependente o sucesso de tratamento que compreende os casos de cura comprovada e os casos de tratamento completo, e como variável independente a exposição a qualquer uma das partes que compõe o benefício de transferência de renda condicionada do PBF. Foram avaliados potenciais confundidores e foram incluídos no modelo multivariado as variáveis com completitude acima de $80 \%$ e significância estatística $(p<0,20)$ na análise bivariada. As variáveis forma clínica e sorologia para HIV foram incluídas devido a constatação da sua associação com desfecho e exposição na literatura independe do critério acima. Foram estimadas as medidas de associação por meio do risco relativo e 
respectivos intervalos de confiança entre a exposição ao benefício do PBF e o sucesso de tratamento da tuberculose por meio do modelo de regressão de Poisson com variância robusta (Newton et al. 2010). Tal modelo é adequado para estimar risco relativo em estudos de coorte de desfechos comuns, como no presente estudo no qual a proporção de cura é superior a 70\% (McNutt et al. 2003). Para examinar uma possível modificação do efeito do PBF no sucesso e tratamento segundo a realização de TDO, também foi aplicado esse mesmo modelo após estratificação da base de dados segundo realização do TDO.

Os dados foram analisados nos softwares Stata v.12 (StataCorp, College Station, Texas USA).

\section{Resultados}

Conforme apresentado no quadro 1 , do total de 71.660 casos novos de TB diagnosticados em 2010, 76,1\% (54.527) não eram cadastrados no Cad.Único, 10,8\% (7.719) eram cadastrados porem não beneficiários do PBF e 13,1\% (9.414) eram cadastrados e beneficiários do PBF.

A população do estudo foi composta por $7.152(79,3 \%)$ casos que foram expostos à transferência de renda durante o tratamento da TB e 1.863 (20,7\%) casos que receberam o beneficio após o tratamento. 
Figura 1. Fluxograma de inclusão na população de estudo

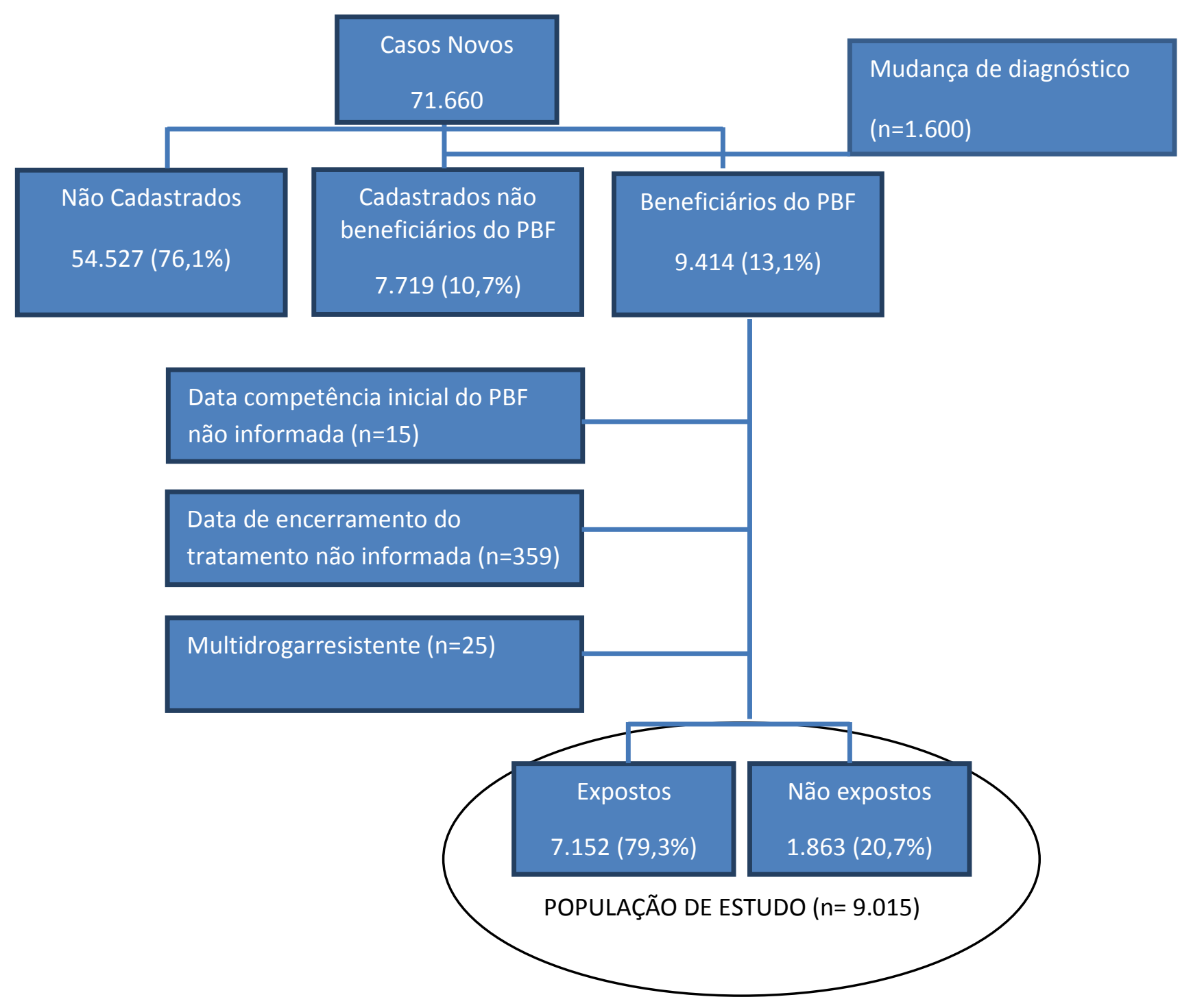

Fonte: Sinan/MS, Cad.Úncio/ MDS, Folha de pagamento PBF/ CEF 
A população de estudo, apresentou o perfil demográfico e clínico e socioeconômico conforme Tabelas 1 e 2 respectivamente. Dentre as características demográficas e clínicas, destaca-se a elevada proporção de casos do sexo feminino na população de estudo, em ambos os grupos exposto e não exposto, em comparação com o perfil demográfico dos casos de TB no país. As variáveis faixa etária, raça/ cor, analfabetismo, status sorológico para HIV e diabetes mellitus apresentaram diferenças significativas entre os grupos exposto e não exposto.

Em relação às características socioeconômicas, todas apresentaram associação com a exposição, sendo a proporção de casos residentes na zona rural e em domicílios com menos de 4 cômodos maior entre os expostos. Além disso, todas as características do domicílio se apresentaram de forma inapropriada em maior proporção entre os grupos dos expostos. 
Tabela 1. Características demográficas e clínicas da população de estudo segundo exposição ao PBF, Brasil, 2010.

\begin{tabular}{|c|c|c|c|c|c|c|c|}
\hline \multicolumn{2}{|c|}{ Características demográficas e clínicas } & \multicolumn{2}{|c|}{$\begin{array}{l}\text { Exposto } \\
\text { durante o } \\
\text { tratamento da } \\
\text { TB }\end{array}$} & \multicolumn{2}{|c|}{$\begin{array}{l}\text { Não exposto } \\
\text { durante o } \\
\text { tratamento da } \\
\text { TB }\end{array}$} & \multirow{2}{*}{$\begin{array}{l}\text { Total } \\
\text { n }\end{array}$} & \multirow[t]{2}{*}{$\begin{array}{l}\text { p } \\
\text { valor*** }\end{array}$} \\
\hline & & $\mathbf{n}$ & $\%$ & $\mathbf{n}$ & $\%$ & & \\
\hline Total & & 7.152 & 100,0 & 1.863 & 100,0 & 9.015 & \\
\hline \multirow{3}{*}{ Sexo } & Feminino & 3.625 & 50,7 & 954 & 51,2 & 4.579 & 0,692 \\
\hline & Masculino & 3.526 & 49,3 & 909 & 48,8 & 4.435 & \\
\hline & Dados faltantes & 1 & 0,0 & 0 & 0,0 & 1 & \\
\hline \multirow{4}{*}{ Faixa etária } & $<15$ anos & 619 & 8,7 & 143 & 7,7 & 762 & 0,050 \\
\hline & 15 a 49 anos & 5.479 & 76,6 & 1.477 & 79,3 & 6.956 & \\
\hline & $>=a 50$ & 1.054 & 14,7 & 243 & 13,0 & 1.297 & \\
\hline & Dados faltantes & 0 & 0,0 & 0 & 0,0 & 0 & \\
\hline \multirow{3}{*}{ Raça/ cor } & Não negro & 1.897 & 26,5 & 545 & 29,3 & 2.442 & 0,012 \\
\hline & Negro* $^{*}$ & 4.727 & 66,1 & 1172 & 62,9 & 5.899 & \\
\hline & Dados faltantes & 528 & 7,4 & 146 & 7,8 & 674 & \\
\hline \multirow{3}{*}{ Forma clínica } & Pulmonar & 6.273 & 87,7 & 1645 & 88,3 & 7.918 & 0,498 \\
\hline & Extrapulmonar & 879 & 12,3 & 218 & 11,7 & 1.097 & \\
\hline & Dados faltantes & 0 & 0,0 & 0 & 0,0 & 0 & \\
\hline \multirow{8}{*}{$\begin{array}{l}\text { Agravos } \\
\text { associados }\end{array}$} & HIV positivo & 484 & 6,77 & 162 & 8,7 & 646 & 0,001 \\
\hline & negativo & 3.576 & 50,0 & 973 & 52,2 & 4.549 & \\
\hline & Sorologia desconhecida & 3.092 & 43,2 & 728 & 39,1 & 3.820 & \\
\hline & $\mathrm{DM}^{* *} \mathrm{Sim}$ & 369 & 5,2 & 71 & 3,8 & 440 & 0,009 \\
\hline & Não & 5.941 & 83,1 & 1.613 & 86,6 & 7.554 & \\
\hline & Dados faltantes & 842 & 11,8 & 179 & 9,6 & 1.021 & \\
\hline & Alcoolismo Sim & 754 & 10,5 & 216 & 11,6 & 970 & 0,293 \\
\hline & Não & 5.623 & 78,6 & 1.477 & 79,3 & 7.100 & \\
\hline
\end{tabular}


$\begin{array}{llllll}\text { Dados faltantes } \quad 775 \quad 10,8 & 170 & 9,1 & 945\end{array}$

*preto+pardo, ${ }^{*} \mathrm{DM}=$ diabetes mellitus ${ }^{* \star \star}$ teste Chi quadrado

Fonte: Sinan/MS, Cad.Úncio/ MDS, Folha de pagamento PBF/ CEF 
Tabela 2. Características socioeconômicas da população de estudo segundo exposição ao PBF, Brasil, 2010.

\begin{tabular}{|c|c|c|c|c|c|c|c|}
\hline \multicolumn{2}{|c|}{ Características socioeconômicas } & \multicolumn{2}{|c|}{$\begin{array}{l}\text { Exposto durante o } \\
\text { tratamento da TB }\end{array}$} & \multicolumn{2}{|c|}{$\begin{array}{l}\text { Não exposto } \\
\text { durante o } \\
\text { tratamento da TB }\end{array}$} & \multirow{2}{*}{$\begin{array}{l}\text { Total } \\
\text { n }\end{array}$} & \multirow[t]{2}{*}{ p valor } \\
\hline & & $\mathbf{n}$ & $\%$ & $\mathbf{n}$ & $\%$ & & \\
\hline Total & & 7.152 & 100,0 & 1.863 & 100,0 & 9.015 & \\
\hline \multirow{3}{*}{ Analfabetismo } & Alfabetizado & 6.043 & 84,5 & 1635 & 87,8 & 7.678 & \\
\hline & Analfabeto & 1.094 & 15,3 & 226 & 12,1 & 1.320 & \\
\hline & Dados faltantes & 15 & 0,2 & 2 & 0,1 & 17 & \\
\hline \multirow{8}{*}{$\begin{array}{l}\text { Características } \\
\text { do domicílio }\end{array}$} & Zona & & & & & & 0,000 \\
\hline & Urbano & 5.952 & 83,2 & 1.608 & 86,3 & 7.560 & \\
\hline & Rural & 1.189 & 16,6 & 224 & 12,0 & 1.413 & \\
\hline & Dados faltantes & 11 & 0,2 & 31 & 1,7 & 42 & \\
\hline & $\begin{array}{l}\text { Quantidade de } \\
\text { cômodos }\end{array}$ & & & & & & 0,000 \\
\hline & 4 ou + cômodos & 2.678 & 37,4 & 571 & 30,7 & 3.249 & \\
\hline & <4 cômodos & 4.474 & 62,6 & 1.292 & 69,4 & 5.766 & \\
\hline & Dados faltantes & 0 & 0,0 & 0 & 0,0 & 0 & \\
\hline
\end{tabular}


Material do piso*

$\begin{array}{llllll}\text { Apropriado } & 2.976 & 41,6 & 1.104 & 59,3 & 4080 \\ \text { Inapropriado } & 4.070 & 56,9 & 651 & 34,9 & 4.72 \\ \text { Dados faltantes } & 108 & 1,5 & 5,8 & 106 & 2014\end{array}$

\begin{tabular}{lllllll} 
Parentesco & & & & & & 0,000 \\
Não parente & 12 & 0,2 & 4 & 0,2 & 16 & \\
Chefe de família & 3166 & 44,3 & 1094 & 58,7 & 4260 & \\
Parente & 3899 & 54,5 & 753 & 40,4 & 4652 & \\
Dados faltantes & 75 & 1,0 & 12 & 0,6 & 87 & \\
\hline $\begin{array}{l}\text { Renda familiar per } \\
\text { capta ** }\end{array}$ & & & & & & 0,006 \\
$\quad$ Mediana & 50,00 & & 50,00 & & 50,00 & \\
Intervalo IQ** & 55,00 & & 60,00 & & 57,00 &
\end{tabular}

*terra, madeira aparelhada e outro material não especificado

**reais brasileiros

***intervalo interquartílico

Fonte: Sinan/MS, Cad.Úncio/ MDS, Folha de pagamento PBF/ CEF 
Em relação ao desfecho de tratamento da tuberculose, a proporção de cura foi $81,7 \%$ entre os expostos ao benefício do PBF durante o tratamento da tuberculose, 5,4 pontos percentuais maior que entre os não expostos durante 0 tratamento da tuberculose que foi de 76,3\%. A média do valor do benefício recebido pela família dos casos de tuberculose foi de 139,00 reais (DP 80,00 reais). A análise multivariada encontrou um risco relativo de 1,07 (CI 95\%; 1,04 - 1,07), que significa um efeito de $7 \%$ do benefício do PBF no aumento do sucesso de tratamento da tuberculose quando ajustada para as covariáveis faixa etária, raça/ cor, diabetes mellitus, sorologia para HIV, forma clínica, tratamento diretamente observado, zona de residência, número de cômodos e material do piso do domicílio, renda familiar per capita e analfabetismo (Tabela 4). 
Tabela 3. Desfecho de tratamento da tuberculose segundo exposição ao PBF, Brasil, 2010.

\section{Cura}

Variáveis

RR (IC 95\%)

\begin{tabular}{llll} 
& Bruto & Ajustado \\
\hline Programa Bolsa Família & $\mathbf{1 , 0 7}(\mathbf{1 , 0 6 - 1 , 5 2 )}$ & $\mathbf{1 , 0 7}$ & $(\mathbf{1 , 0 4 - 1 , 1 1 )}$ \\
$<\mathbf{1 5}$ anos & 1 & \\
$\mathbf{1 5 - 5 0}$ anos & 0,94 & $(0,90-0,97)$ \\
$>\mathbf{5 0}$ anos & 0,95 & $(0,91-0,99)$ \\
Negros & & 0,95 & $(0,93-0,97)$ \\
Diabetes mellittus & 0,99 & $(0,95-1,04)$ \\
HIV negativo & 1 & \\
HIV positivo & 0,78 & $(0,73-0,82)$ \\
Sorologia HIV desconhecida & 0,92 & $(0,90-0,94)$ \\
Forma extrapulmonar & 1,01 & $(0,97-1,04)$ \\
TDO não realizado & 0,88 & $(0,86-0,90)$ \\
Área rural & 0,98 & $(0,96-1,02)$ \\
Número de cômodos $<4$ & 1,00 & $(0,98-1,02)$ \\
Piso do domicílio inapropriado & & & \\
Renda & 0,96 & $(0,94-0,99)$ \\
Analfabeto & 0,97 & $(0,95-1,00)$ \\
& 0,98 & $(0,95-0,99)$
\end{tabular}

**pretos e pardos

***terra, madeira aparelhada e outro material não especificado

Fonte: Sinan/MS, Cad.Úncio/ MDS, Folha de pagamento PBF/ CEF 
Em análise estratificada segundo realização do tratamento diretamente observado, pode-se perceber que para os casos que realizaram tratamento auto administrado o efeito do benefício do PBF na cura foi maior. O risco relativo ajustado pelas mesmas covariáveis mencionadas na análise não estratificada mostrou um efeito de $5 \%$ do benefício do PBF na cura da tuberculose entre os pacientes sobre TDO em comparação a 11\% entre os que não realizaram conforme tabela 5 . 
Tabela 4. Desfecho de tratamento da tuberculose segundo exposição ao PBF estratificado por realização do tratamento diretamente observado. Brasil, 2010.

\begin{tabular}{|c|c|c|c|c|c|c|c|}
\hline \multirow{2}{*}{$\begin{array}{l}\text { Programa Bolsa } \\
\text { Família* }\end{array}$} & \multicolumn{2}{|l|}{ Cura } & \multicolumn{2}{|c|}{ Não cura* } & \multirow{2}{*}{ Total } & \multicolumn{2}{|l|}{ RR (IC 95\%) } \\
\hline & $n$ & $\%$ & $\mathbf{n}$ & $\%$ & & Bruto & Ajustado** \\
\hline \multicolumn{8}{|c|}{ Tratamento diretamente observado } \\
\hline $\begin{array}{l}\text { Exposto durante o } \\
\text { tratamento da TB }\end{array}$ & 3.066 & 80,7 & 472 & 19,3 & 3.538 & $1,04(1,00-1,57)$ & $1,05(1,01-1,08)$ \\
\hline $\begin{array}{l}\text { Não exposto } \\
\text { durante o } \\
\text { tratamento da TB }\end{array}$ & 734 & 76,6 & 144 & 23,4 & 878 & & \\
\hline Total & 3.800 & 80 & 616 & 19,9 & 4.416 & & \\
\hline
\end{tabular}

Tratamento auto administrado

\begin{tabular}{|c|c|c|c|c|c|c|c|}
\hline $\begin{array}{l}\text { Exposto durante o } \\
\text { tratamento da TB }\end{array}$ & 2.774 & 80,0 & 840 & 19,6 & 3.614 & $1,10(1,05-1,15)$ & $1,11(1,05-1,16)$ \\
\hline $\begin{array}{l}\text { Não exposto } \\
\text { durante o } \\
\text { tratamento da TB }\end{array}$ & 687 & 73,8 & 298 & 26,2 & 985 & & \\
\hline Total & 3.461 & 78,6 & 1.138 & 21,4 & 4.599 & & \\
\hline
\end{tabular}


*abandono, óbito, transferência e ignorado

**ajustado por: faixa etária, raça/ cor, diabetes mellitus, sorologia para HIV, forma clínica, zona de

residência, número de cômodos, material do piso do domicílio, renda familiar per capta e analfabetismo

Fonte: Sinan/MS, Cad.Úncio/ MDS, Folha de pagamento PBF/ CEF 


\section{Discussão}

Os resultados deste estudo estimam a magnitude de um programa de transferência de renda condicionada pode aumentar a proporção de cura dos pacientes com tuberculose. Este estudo, realizado com a totalidade de pacientes com tuberculose no Brasil elegíveis para o Programa Bolsa Família, um dos maiores programas de transferência de renda condicionada do mundo, é relevante porque o sucesso de tratamento continua sendo um grande desafio para o país. Elevadas taxas de abandono de tratamento e sistema de informação frágil no acompanhamento dos casos (BRASIL 2014b) dificultam o atingimento da meta recomendada pelo OMS de $85 \%$ de cura dos casos novos de tuberculose (WHO 2006). Os progressos nos determinantes sociais da saúde alcançados com o PBF têm tido um efeito na saúde dos brasileiros(Paim et al. 2011), contribuindo assim para o controle de doenças infecciosas como a tuberculose no país.

Sabe-se que enquanto a Estratégia DOTS contribui, significativamente, na redução da prevalência e da mortalidade por tuberculose, o desenvolvimento socioeconômico continua sendo o principal fator associado à queda da incidência da doença em muitos cenários (Boccia et al. 2011). Em revisão sistemática, programas de transferência de renda e microcrédito mostraram influencia em fatores de risco para TB em países de alta carga da doença. Em princípio tais intervenções tem o potencial de melhorar o acesso da população a serviços de saúde e reduzir a vulnerabilidade ao adoecimento por TB por meio da melhoria das condições socioeconômicas das famílias e da segurança alimentar em termos de acesso a alimentos e cardápio diversificado (Boccia et al. 2011). Apesar da literatura sobre o efeito de intervenções de proteção social na tuberculose em si ainda ser muito escassa, as experiências revisadas corroboram a hipótese da efetividade de intervenções sociais combinadas a ações programáticas de controle da tuberculose. Por meio das condições materiais, circunstancias psicossociais e acesso e procura a serviços de saúde, essas intervenções tem não só o potencial de reduzir a 
vulnerabilidade ao adoecimento por TB, mas também de melhorar o acesso a tratamento de qualidade da TB.

Nesse sentido, a efetividade de $7 \%$ do PBF no sucesso de tratamento da tuberculose é um importante resultado e representa a possibilidade de mais uma estratégia no seu enfrentamento, visto que se trata de uma doença multifatorial que exige abordagens intersetoriais. Além disso, o PBF não é específico para pacientes com tuberculose, indicando que o desenho adequado de um benefício para essa população alvo específica e com condicionalidades na adesão ao tratamento pode potencializar os resultados. O resultado da análise estratificada pelo TDO aponta que a transferência de renda pode ser uma estratégia complementar a essa ou até mesmo uma alternativa para adesão entre os pacientes em tratamento auto administrado.

Publicação sobre um estudo de intervenções socioeconômicas para o controle da tuberculose, realizado no Peru com amostra de casos muito menor, mostra resultados semelhantes (Rocha et al. 2011). A proporção de sucesso de tratamento foi $97 \%$ entre os pacientes que aderiram a intervenções socioeconômicas, incluindo transferência de renda condicionada, representando um aumento de 6\% em relação à linha de base calculada, sendo estatisticamente significativo (Rocha et al. 2011). Estudo similar conduzido na África do Sul não encontrou efeito da transferência de renda na cura da tuberculose. Contudo apresentou importante limitação no atrasado do pagamento do benefício aos pacientes, e encontrou associação entre o tempo de recebimento do benefício com o sucesso de tratamento (Lutge et al. 2013).

A Organização Pan-americana de Saúde (OPAS), em informe da reunião de coordenadores de programas nacionais de controle da tuberculose, documenta a experiência do Equador, Brasil e Colômbia no que tange a introdução da tuberculose nos programas de proteção social. No caso do programa de controle da tuberculose do Equador, após um ano de implementação do benefício social para os pacientes com tuberculose drogaresistente (TBDR) houve melhora da adesão ao tratamento, e a proporção de abandono de TBDR diminuiu para 5\%. Apesar de a 
intervenção ser voltada para outra população alvo mais restrita, os resultados corroboram o presente estudo. Desafios semelhantes também foram citados, como reduzir o atraso na transferência do benefício e sensibilizar os gestores para sustentabilidade da iniciativa (OPAS 2012).

Uma limitação do presente estudo é que a população de estudo não representa o total de casos novos de tuberculose do país. Representa os pacientes pertencentes a famílias em vulnerabilidade social segundo critérios de renda e composição familiar. O fato de apenas aproximadamente $24 \%$ de casos novos de TB estarem cadastrados no Cad.Único e apenas em torno de $14 \%$ serem beneficiários do PBF poderia estar associado ao método de pareamento das bases de dados em si, ou a própria característica da população cadastrada e beneficiária do PBF, que apresenta elevada proporção de mulheres e criança, enquanto que a maioria dos casos de tuberculose do país são do sexo masculino. Contudo, a utilização como grupo de comparação de casos elegíveis ao PBF, que podem representar fidedignamente contrafactuais, assegura que as estimativas de avaliação de impacto não foram enviesadas. As diferencias estatisticamente significativas encontradas para algumas variáveis são influenciadas pelo tamanho da amostra grande que gera um elevado poder estadístico.

Além disso, nem toda a vulnerabilidade social é captada pelos critérios de renda e composição familiar para elegibilidade no PBF. A pobreza também está relacionada ao adoecimento por tuberculose que possui forte determinação social, e assim pode-se aventar a hipótese que grande partedo restante dos casos poderiam se beneficiar de estratégias de proteção social conforme o resultado encontrado na população de estudo.

No Brasil, o acesso e adesão ao tratamento da tuberculose vêm sendo trabalhado principalmente por meio do fortalecimento da descentralização das ações de controle da doença para a atenção básica. A estratégia do tratamento diretamente observado e a adoção do tratamento com dose fixa combinada, foram os principais avanços obtidos nos últimos anos para a adesão ao tratamento(BRASIL 2014b). Contudo, apesar do tratamento da tuberculose ser garantido pelo SUS, o tratamento da doença 
resulta em custos diretos médicos e não médicos e indiretos relacionados com a redução da capacidade de gerar renda durante o período de adoecimento (Mauch et al. 2013).Por ser uma doença infecciosa que se comportam de forma crônica, os custos são acentuados pelo tratamento de longa duração, e isso impacta de forma mais significativa nos pacientes em situação de vulnerabilidade social.

Assim, para atingir a eliminação da doença proposta pela nova estratégia de controle da TB pós 2015, os programas de controle da tuberculose necessitam de estratégias inovadoras (Boccia et al. 2011). O presente estudo mostrou a efetividade de intervenções sociais combinadas a ações programáticas de controle da tuberculose no Brasil. Nesse sentido, estratégias como a transferência de renda condicionada específicas para os pacientes com tuberculose, representam uma possibilidade para o controle da tuberculose e devem ser adotadas em países com cenários semelhantes.

\section{Referências}

Adato, M. \& Hoddinott, J., 2008. Social Protection: Opportunities for Africa. , (September).

Blas, E. \& Kurup, A.S., 2010. Equity, social determinants and public health programmes. Who, p.77ff.

Boccia, D. et al., 2011. UKPMC Funders Group Cash transfer and microfinance interventions for tuberculosis control : review of the impact evidence and policy implications. International Journal of Tuberculosis and Lung Disease, 15(Suppl 2), pp.37-49.

BRASIL, 2012. Boletim Epidemiológico: Especial Tuberculose. Boletim Epidemiológico, 43, p.12.

BRASIL, 2015a. Data Social. DATA SOCIAL. Available at: http://aplicacoes.mds.gov.br/sagidata/METRO/metro_ds.php?p_id=293.

BRASIL, 2014a. Guia de vigilância em saúde, Ministério da Saúde.

BRASIL, 2015b. Inquérito de disponibilização de Benefício para Pacientes com Tuberculose nod 181 Municípios Prioritários para o Controle da Tuberculose,

BRASIL, 2009. Manual de Orientações do Bolsa Família na Saúde, Ministério da Saúde. 
BRASIL, 2014b. O controle da tuberculose no Brasil: avanços, inovações e desafios. , 44.

BRASIL, 2011. Resolução $n^{\circ} 444$, de 6 de julho de 2011. A resolução que trata do enfrentamento da tuberculose no Brasil, CONSELHO NACIONAL DE SAÚDE.

Hanson, C., 2002. Tuberculosis, poverty and inequity: a review of the literature and discussion of issues.

Hargreaves, J.R. et al., 2011. The social determinants of tuberculosis: from evidence to action. American journal of public health, 101(4), pp.654-662.

Jaramillo E, 1999. Encompassing treatment with prevention: the path for a lasting control of tuberculosis. Social Science and Medicine, 49, pp.393-404.

Lienhardt, C., 2001. From exposure to disease: the role of environmental factors in susceptibility to and development of tuberculosis. Epidemiologic reviews, 23(2), pp.288-301.

Lindert, K. et al., 2007. The Nuts and Bolts of Brazil's Bolsa Família Program: Implementing Conditional Cash Transfers in a Decentralized Context. Social Protection Discussion Paper Series, (0709), p.144.

Lönnroth, K. et al., 2010. Tuberculosis control and elimination 2010-50: cure, care, and social development. The Lancet, 375(9728), pp.1814-1829. Available at: http://dx.doi.org/10.1016/S0140-6736(10)60483-7.

Lutge, E. et al., 2013. Economic support to improve tuberculosis treatment outcomes in South Africa: a pragmatic cluster-randomized controlled trial. Trials, 14(1), p.154. Available at: http://www.pubmedcentral.nih.gov/articlerender.fcgi?artid=3680200\&tool=pmcentre $z \&$ rendertype $=$ abstract.

Mauch, V. et al., 2013. Free tuberculosis diagnosis and treatment are not enough :Int J Tuberc Lung Dis, 17(October 2012), pp.381-387.

McNutt, L.A. et al., 2003. Estimating the relative risk in cohort studies and clinical trials of common outcomes. American Journal of Epidemiology, 157(10), pp.940-943.

Newton, H.J. et al., 2010. The Stata Journal. Stata Journal, 10, pp.288-308. Available at: http://ideas.repec.org/a/tsj/stataj/v7y2007i4p465-506.html.

OPAS, 2012. INFORME DE LA REUNIÓN REGIONAL DE JEFES DE PROGRAMAS NACIONALES DE CONTROL DE LA TUBERCULOSIS,

Paim, J. et al., 2011. The Brazilian health system: History, advances, and challenges. The Lancet, 377(9779), pp.1778-1797. Available at: http://dx.doi.org/10.1016/S01406736(11)60054-8. 
Rocha, C. et al., 2011. The Innovative Socio-economic Interventions Against Tuberculosis (ISIAT) project: An operational assessment. International Journal of Tuberculosis and Lung Disease, 15(Suppl 2).

Savioli, L., Daumerie, D. \& Who, F., 2010. First WHO report on neglected tropical diseases: working to overcome the global impact of neglected tropical diseases. World Health Organization, pp.1-184.

Soares, S. \& Sátyro, N., 2009. O Programa Bolsa Família: Desenho Institucional, Impactos E Possibilidades Futuras. , p.41. Available at:

http://www.ipea.gov.br/agencia/images/stories/PDFs/TDs/td_1424.pdf.

WHO, 1998. Cid-1O, Organização Mundial de Saúde.

WHO, 2015. Global strategy and targets for tuberculosis prevention, care and control after 2015. , pp.1-23. Available at: http://www.who.int/tb/post2015_TBstrategy.pdf?ua=1.

WHO, 2014a. Global Tuberculosis Report 2014,

WHO, 2014b. Global Tuberculosis Report, country profile,

WHO, 2006. The Stop TB Strategy. , pp.1-20. Available at:

http://whqlibdoc.who.int/hq/2006/WHO_HTM_STB_2006.368_eng.pdf.

World Bank, 2010. Handbook on ImpactEvaluation: quantitative methods and practices,

Ximenes, R. a D. a et al., 2009. Is it better to be rich in a poor area or poor in a rich area? A multilevel analysis of a case-control study of social determinants of tuberculosis. International Journal of Epidemiology, 38, pp.1285-1294.

\section{DISCUSSÃO E CONSIDERAÇÕES FINAIS}

Os resultados deste estudo mostram como um programa de transferência de renda condicionada pode aumentar a proporção de cura dos pacientes com tuberculose. Este estudo, realizado com a totalidade de pacientes com tuberculose no Brasil elegíveis para o Programa Bolsa Família, um dos maiores programas de transferência de renda condicionada do mundo, é relevante porque o sucesso de tratamento continua sendo um grande desafio para o país. Elevadas taxas de abandono de tratamento e sistema de informação frágil no acompanhamento dos casos (BRASIL 2014b) dificultam o atingimento da meta recomendada pelo OMS de 85\% de 
cura dos casos novos de tuberculose (WHO 2006). Os progressos nos determinantes sociais da saúde alcançados com o PBF têm tido um efeito na saúde dos brasileiros (Paim et al. 2011), contribuindo assim para o controle de doenças infecciosas como a tuberculose no país.

A proporção de cura da população de estudo, em ambos os grupos exposto e não exposto, foi maior que a registrada no país para esse indicador de 72\% em 2010 (WHO 2014b). Isso pode estar associado às condicionalidades do PBF, que apesar de não serem específicas para tuberculose, promovem uma aproximação da família com os serviços de saúde, uma vez que toda família é vinculada a uma unidade básica de saúde para o acompanhamento das condicionalidades do PBF (BRASIL 2009).

Sabe-se que enquanto a Estratégia DOTS contribui, significativamente, na redução da prevalência e da mortalidade por tuberculose, o desenvolvimento socioeconômico continua sendo o principal fator associado à queda da incidência da doença em muitos cenários (Boccia et al. 2011). Em revisão sistemática, programas de transferência de renda e microcrédito mostraram influencia em fatores de risco para TB em países de alta carga da doença. Em princípio tais intervenções tem o potencial de melhorar o acesso da população a serviços de saúde e reduzir a vulnerabilidade ao adoecimento por TB por meio da melhoria das condições socioeconômicas das famílias e da segurança alimentar em termos de acesso a alimentos e cardápio diversificado (Boccia et al. 2011).Apesar da literatura sobre o efeito de intervenções de proteção social na tuberculose em si ainda ser muito escassa, as experiências revisadas corroboram a hipótese da efetividade de intervenções sociais combinadas a ações programáticas de controle da tuberculose. Por meio das condições materiais, circunstancias psicossociais e acesso e procura a serviços de saúde, essas intervenções tem não só o potencial de reduzir a vulnerabilidade ao adoecimento por TB, mas também de melhorar o acesso a tratamento de qualidade da TB.

Nesse sentido, a efetividade de $7 \%$ do PBF no sucesso de tratamento da tuberculose é um importante resultado e representa a possibilidade de 
mais uma estratégia no seu enfrentamento, visto que se trata de uma doença multifatorial que exige abordagens intersetoriais. Além disso, o PBF não é específico para pacientes com tuberculose, indicando que o desenho adequado de um benefício para essa população alvo específica e com condicionalidades na adesão ao tratamento pode potencializar os resultados. O resultado da análise estratificada pelo TDO aponta que a transferência de renda pode ser uma estratégia complementar a essa ou até mesmo uma alternativa para adesão entre os pacientes em tratamento auto administrado.

Publicação sobre um estudo de intervenções socioeconômicas para o controle da tuberculose, realizado no Peru com amostra de casos muito menor, mostra resultados semelhantes (Rocha et al. 2011). A proporção de sucesso de tratamento foi $97 \%$ entre os pacientes que aderiram a intervenções socioeconômicas, incluindo transferência de renda condicionada, representando um aumento de 6\% em relação à linha de base calculada, sendo estatisticamente significativo (Rocha et al. 2011). Estudo similar conduzido na África do Sul não encontrou efeito da transferência de renda na cura da tuberculose. Contudo apresentou importante limitação no atrasado do pagamento do benefício aos pacientes, e encontrou associação entre o tempo de recebimento do benefício com o sucesso de tratamento (Lutge et al. 2013).

A Organização Pan-americana de Saúde (OPAS), em informe da reunião de coordenadores de programas nacionais de controle da tuberculose, documenta a experiência do Equador, Brasil e Colômbia no que tange a introdução da tuberculose nos programas de proteção social. No caso do programa de controle da tuberculose do Equador, após um ano de implementação do benefício social para os pacientes com tuberculose drogaresistente (TBDR) houve melhora da adesão ao tratamento, e a proporção de abandono de TBDR diminuiu para 5\%. Apesar de a intervenção ser voltada para outra população alvo mais restrita, os resultados corroboram o presente estudo. Desafios semelhantes também foram citados, como reduzir o atraso na transferência do benefício e sensibilizar os gestores para sustentabilidade da iniciativa (OPAS 2012). 
Uma limitação do estudo é que a população de estudo não representa o total de casos novos de tuberculose do país. Representa os pacientes pertencentes a famílias em vulnerabilidade social segundo critérios de renda e composição familiar. O fato de apenas aproximadamente $24 \%$ de casos novos de TB estarem cadastrados no Cad.Único e apenas em torno de $14 \%$ serem beneficiários do PBF poderia estar associado ao método de pareamento das bases de dados em si, ou a própria característica do PBF que cadastra prioritariamente mulheres como beneficiárias chefes de família, enquanto que a maioria dos casos de tuberculose do país são do sexo masculino. Contudo, a utilização como grupo de comparação de casos elegíveis ao PBF, que podem representar fidedignamente contrafactuais, assegura que as estimativas de avaliação de impacto não foram enviesadas. As diferencias estatisticamente significativas encontradas para algumas variáveis é influenciadas pelo tamanho da amostra grande que gera um elevado poder estadístico.

Além disso, nem toda a vulnerabilidade social é captada pelos critérios de renda e composição familiar para elegibilidade no PBF. A pobreza também está relacionada ao adoecimento por tuberculose que possui forte determinação social, e assim pode-se aventar a hipótese que grande parte do restante dos casos poderiam se beneficiar de estratégias de proteção social conforme o resultado encontrado na população de estudo.

No Brasil, o acesso e adesão ao tratamento da tuberculose vêm sendo trabalhado principalmente por meio do fortalecimento da descentralização das ações de controle da doença para a atenção básica. $A$ estratégia do tratamento diretamente observado, que consiste na tomada observada da medicação por um profissional de saúde ou pessoa orientada por pelo menos três vezes na semana na fase de ataque (2 primeiros meses) e duas na fase de manutenção do tratamento (BRASIL 2014a) e a adoção do tratamento com dose fixa combinada, que permitiram uma redução na quantidade de comprimidos a ser ingerida diariamente, foram os principais avanços obtidos nos últimos anos para a adesão ao tratamento (BRASIL 2014b). Atualmente outras estratégias de adesão estão sendo 
incorporadas pelos programas de controle da tuberculose, como o acolhimento humanizado, o projeto terapêutico singular, os incentivos de adesão ao tratamento, e os benefícios sociais de proteção social (BRASIL 2015b).

Contudo, apesar do tratamento da tuberculose ser garantido pelo SUS, há custos diretos relacionados ao deslocamento aos serviços de saúde e indiretos devido a redução da capacidade de gerar renda durante o período da doença. Por ser uma doença infecciosa que se comportam de forma crônica, os custos são acentuados pelo tratamento de longa duração, e isso impacta de forma mais significativa nos pacientes em situação de vulnerabilidade social. Estudo sobre custos realizado em três diferentes países de diferentes regiões mostrou que os custos diretos médicos e não médicos dos pacientes com diagnóstico e tratamento de tuberculose representam apenas de 12 a $27 \%$ dos custos totais. Esses estão relacionados a testes e medicamentos complementares ao diagnóstico e tratamento da TB, a transporte para consultas de acompanhamento, a alimentação e acomodação quando do atendimento em outro local que o de residência. Contudo, a maior parte dos custos é devido à perda de renda durante o adoecimento. Os resultados mostraram que 27 a $70 \%$ dos pacientes interromperam sua atividade produtiva devido à tuberculose e a redução de renda chegou até 82,9\% (Mauch et al. 2013).

Assim, para atingir a eliminação da doença proposta pela nova estratégia de controle da TB pós 2015, os programas de controle da tuberculose necessitam de estratégias inovadoras (Boccia et al. 2011). O presente estudo mostrou a efetividade de intervenções sociais combinadas a ações programáticas de controle da tuberculose no Brasil. Nesse sentido, estratégias como a transferência de renda condicionada específicas para os pacientes com tuberculose, representam uma possibilidade para o controle da tuberculose e devem ser adotadas em países com cenários semelhantes.

\section{REFERÊNCIAS}


Adato, M. \& Hoddinott, J., 2008. Social Protection: Opportunities for Africa. , (September).

Blas, E. \& Kurup, A.S., 2010. Equity, social determinants and public health programmes. Who, p.77ff.

Boccia, D. et al., 2011. UKPMC Funders Group Cash transfer and microfinance interventions for tuberculosis control : review of the impact evidence and policy implications. International Journal of Tuberculosis and Lung Disease, 15(Suppl 2), pp.37-49.

BRASIL, 2012. Boletim Epidemiológico: Especial Tuberculose. Boletim Epidemiológico, 43, p.12.

BRASIL, 2015a. Data Social. DATA SOCIAL. Available at: http://aplicacoes.mds.gov.br/sagidata/METRO/metro_ds.php?p_id=293.

BRASIL, 2014a. Guia de vigilância em saúde, Ministério da Saúde.

BRASIL, 2015b. Inquérito de disponibilização de Benefício para Pacientes com Tuberculose nod 181 Municípios Prioritários para o Controle da Tuberculose,

BRASIL, 2009. Manual de Orientações do Bolsa Família na Saúde, Ministério da Saúde.

BRASIL, 2014b. O controle da tuberculose no Brasil: avanços, inovações e desafios. , 44.

BRASIL, 2011. Resolução $n^{\circ} 444$, de 6 de julho de 2011. A resolução que trata do enfrentamento da tuberculose no Brasil, CONSELHO NACIONAL DE SAÚDE.

Hanson, C., 2002. Tuberculosis, poverty and inequity: a review of the literature and discussion of issues.

Hargreaves, J.R. et al., 2011. The social determinants of tuberculosis: from evidence to action. American journal of public health, 101(4), pp.654-662.

Jaramillo E, 1999. Encompassing treatment with prevention: the path for a lasting control of tuberculosis. Social Science and Medicine, 49, pp.393-404.

Lienhardt, C., 2001. From exposure to disease: the role of environmental factors in susceptibility to and development of tuberculosis. Epidemiologic reviews, 23(2), pp.288-301.

Lindert, K. et al., 2007. The Nuts and Bolts of Brazil's Bolsa Família Program: Implementing Conditional Cash Transfers in a Decentralized Context. Social Protection Discussion Paper Series, (0709), p.144.

Lönnroth, K. et al., 2010. Tuberculosis control and elimination 2010-50: cure, care, and social development. The Lancet, 375(9728), pp.1814-1829. Available at: http://dx.doi.org/10.1016/S0140-6736(10)60483-7. 
Lutge, E. et al., 2013. Economic support to improve tuberculosis treatment outcomes in South Africa: a pragmatic cluster-randomized controlled trial. Trials, 14(1), p.154. Available at:

http://www.pubmedcentral.nih.gov/articlerender.fcgi?artid=3680200\&tool=pmcentre z\&rendertype=abstract.

Mauch, V. et al., 2013. Free tuberculosis diagnosis and treatment are not enough : Int J Tuberc Lung Dis, 17(October 2012), pp.381-387.

McNutt, L.A. et al., 2003. Estimating the relative risk in cohort studies and clinical trials of common outcomes. American Journal of Epidemiology, 157(10), pp.940-943.

Newton, H.J. et al., 2010. The Stata Journal. Stata Journal, 10, pp.288-308. Available at: http://ideas.repec.org/a/tsj/stataj/v7y2007i4p465-506.html.

OPAS, 2012. INFORME DE LA REUNIÓN REGIONAL DE JEFES DE PROGRAMAS NACIONALES DE CONTROL DE LA TUBERCULOSIS,

Paim, J. et al., 2011. The Brazilian health system: History, advances, and challenges. The Lancet, 377(9779), pp.1778-1797. Available at: http://dx.doi.org/10.1016/S01406736(11)60054-8.

Rocha, C. et al., 2011. The Innovative Socio-economic Interventions Against Tuberculosis (ISIAT) project: An operational assessment. International Journal of Tuberculosis and Lung Disease, 15(Suppl 2).

Savioli, L., Daumerie, D. \& Who, F., 2010. First WHO report on neglected tropical diseases: working to overcome the global impact of neglected tropical diseases. World Health Organization, pp.1-184.

Soares, S. \& Sátyro, N., 2009. O Programa Bolsa Família: Desenho Institucional, Impactos E Possibilidades Futuras. , p.41. Available at:

http://www.ipea.gov.br/agencia/images/stories/PDFs/TDs/td_1424.pdf.

WHO, 1998. Cid-1O, Organização Mundial de Saúde.

WHO, 2015. Global strategy and targets for tuberculosis prevention, care and control after 2015. , pp.1-23. Available at: http://www.who.int/tb/post2015_TBstrategy.pdf?ua=1.

WHO, 2014a. Global Tuberculosis Report 2014,

WHO, 2014b. Global Tuberculosis Report, country profile,

WHO, 2006. The Stop TB Strategy. , pp.1-20. Available at: http://whqlibdoc.who.int/hq/2006/WHO_HTM_STB_2006.368_eng.pdf.

World Bank, 2010. Handbook on ImpactEvaluation: quantitative methods and practices, 
Ximenes, R. a D. a et al., 2009. Is it better to be rich in a poor area or poor in a rich area? A multilevel analysis of a case-control study of social determinants of tuberculosis. International Journal of Epidemiology, 38, pp.1285-1294.

\section{ANEXOS}

Anexo l: Aprovação no Comitê de Ética 


\section{Anexo I: Aprovação no Comitê de Ética}

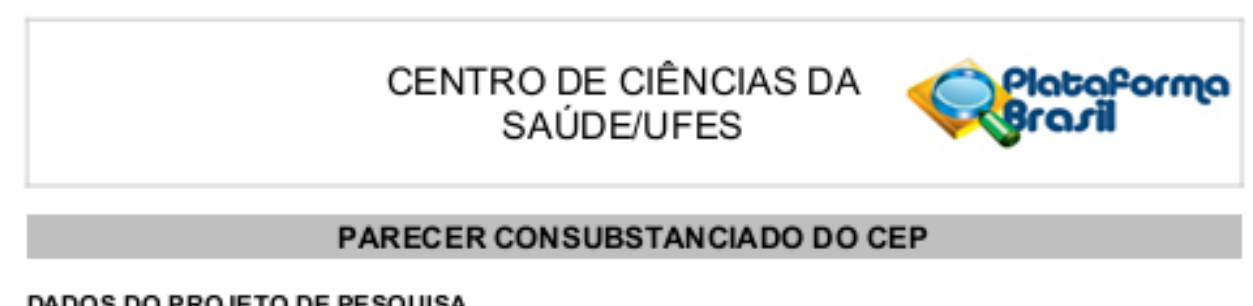

\section{DADOS DO PROJETO DE PESQUISA}

Titulo da Pesquisa: Análise do efeito independente do suporte social na adesāo e nas taxas de sucesso do tratamento de tuberculose em capitais do Brasil

Pesquisador: Ethel Leonor Noia Maciel

Área Temática:

Versāo: 2

CAAE: 12622413.6 .0000 .5060

Instituiçāo Proponente: Centro de Ciências da Saúde ((CCS-UFES))

Patrocinador Principal: Secretaria de Vigilância em Saúde ((SVS/MS))

DADOS DO PARECER

Número do Parecer: 242.831

Data da Relatoria: 10/04/2013

Apresentaçāo do Projeto:

Para a avaliação do recebimento de beneficios sociais e econômicos no tratamento da TB em individuos adultos acompanhados em unidades de saúde, es te estudo foi dividido em três etapas:Primeira etapa: Estudo piloto: Esta etapa terá por objetivo testar os instrumentos do estudo e conhecer operfil dos beneficiários em programa governamentais de transferência de crédito. Primeiramente, um estudo com 15 pacientes será realizado na cidade de Vila-Velha para avaliar a adequaçāo dos instrumentos e fluxograma deseguimento do estudo. Depois será feito um linkage de banco de dados entre o SINAN-TB e o cadastro de pessoas beneficiárias nos programa do Ministério de desenvolvimento social. Um estudo de casocontrole baseado em dados secundários será realizado utilizando os registros do Sinan para os anos 2010 a 2012, e que já fora pareados ao regis to CadÚnico por meio de uma metodologia de pareamento probabilistico desenvolvida no Departamento de Análise da Situaçâo de Saúde. Segunda etapa:Trata-se de um estudo de coorte concorrente (prospectiva). No grupo de expostos serâo considerados os pacientes com TB que recebem algum beneficio, direto ou indireto, de órgāos governamentais e nẫo governamentais. Foram considerados três grupos de exposiçẫ:1. Pacientes com tuberculose e que recebem beneficios diretos foram considerados aqueles onde há algum ganho financeiro para compor a renda do paciente.2.Pacientes com tuberculose e que recebem beneficios indiretos foram considerados aqueles onde há algum ganho indireto,como cesta básica,valetransporte,etc.3. Pacientes com tuberculose e que recebem beneficios indiretos e diretos. Já para o grupo de não

Endereço: Av. Marechá Campos 1468 Bairro: SiN

Bairro: 


\section{CENTRO DE CIÊNCIAS DA SAÚDE/UFES}

expostos serẩo considerados aqueles que nāo recebem qualquer beneficio (direto ou indireto). Terceira etapa:Trata-se de um ensaio randomizado por aglomerados (clusters),

conduzido de forma não cega para a alocaçẫo exposiçẫ. No entanto, a análise será feita por pesquisador cego para a exposiçāo.Serâo criados códigos identificadores para cada grupo o pesquisador responsável pela análise cega nāo terá acesso a estes códigos. Ensaios clinicos por aglomerado (clusters) sāo úteis quando a intervençāo nāo pode ser dissociada do grupo de individuos por questōes feóricas, práticas, ou mesmo éticas. Em relaçẫo às questôes teóricas e práticas, utiliza-se quando a intervençāo que será aplicada pode afetar não apenas um individuo, mas todo um grupo de pessoas (como por exemplo, pessoas que frequentam a mesma unidade de saúde).

\section{Objetivo da Pesquisa:}

Comparar as taxas de sucesso e de abandono do tratamento da TB, segundo os grupos de beneficiários ou nâo do sistema de proteçāo social.

Identificar perfil epidemiológico de pacientes com TB beneficiários do sistema de proteção social do Ministério de Desenvolvimento Social através de relacionamento (linkage) de banco de dados SINAN-TB e cadastro único do Ministério de Desenvolvimento Social. Identificar os fatores dos eixos de vulnerabilidade individual ou comportamental (eixo 1), programática ou insfitucional (eixo 2) e social ou contextual (eixo 3)de individuos com tuberculose, beneficiários ou nâo do sistema de proteção social, e sua relação com os desfechos do tratamento da TB nas capitais brasileiras.

\section{Avaliaçāo dos Riscos e Beneficios:}

Não há nenhum tipo de risco para os participantes da pesquisa

Os beneficios é a possivel identificaçâo se o suporte social tem uma correlaçẫo favoravel com o tratamento da tuberculose.

\section{Comentários e Consideraçōes sobre a Pesquisa:}

Este estudo será desenvolvido em três etapas:(1)Estudo-Piloto a ser realizado no municipio de Vilavelha,ES. Este municipio foi escolhido pela viabilidade operacional da

coordenaçấo do estudo e pelas caracteristicas de proximidade com a capital Vitória para testar os instrumentos. Nesta etapa também será realizado o relacionamento (linkage) dos bancos de dados SINANTB e cadastro único do Ministério de Desenvolvimento Social.

Na segunda etapa, está prevista a instituiçấo do estudo observacional de coorte prospectiva nas seguintes capitais: Manaus, Joāo Pessoa, Salvador, Campo Grande, Rio de Janeiro, Vitória, Curitiba e Porto Alegre e no Distrito Federal, Brasilia. Na terceira etapa, será realizado um ensaio

Endereço: Av. Marechal Campos 1468

Bairro: SiN

CEP: $29040-091$

UF: ES Municipio: VITORIA

Telefone: (27)3335-7211

Email: cepufesighotmailcom; cepgocs.ufes br 


\section{CENTRO DE CIÊNCIAS DA Platoformo SAÚDE/UFES}

randomizado por aglomerados(clusters), conduzido de forma nấo cega, onde serâo avaliados os desfechos do suporte social através da cesta bésica em individuos adultos com TB,tratados em unidades de saúde das seguintes capitais: Manaus, Campo Grande, Rio de Janeiro, Porto Alegre e no Distrito Federal, Brasilia.

Consideraçōes sobre os Termos de apresentaçāo obrigatória:

Folha de Rosto adequada.

TCLE de acordo com as norma da resolução 196CNS.

Cronograma adequado com a data do CEP.

Recomendaçōes:

Acertadas as recomendaçōes propostas.

Conclusōes ou Pendências e Lista de Inadequaçōes:

Sem pendências

Situaçāo do Parecer:

Aprovado

Necessita Apreciaçāo da CONEP:

Năo

Consideraçōes Finais a critério do CEP:

VITORIA, 10 de Abril de 2013

Assinador por:

DANIELLE CABRINI MATTOS

(Coordenador)

Endereço: Av. Marechal Campos 146

Bairro: SIN

UF: ES Municipio: VITORIA

CEP: $29040-091$

Telefone: (27)3335-7211

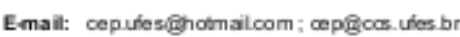

